\title{
ALGEBRAIC SEMANTICS
}

\author{
Jim Thatcher \\ IBM Thomas J. Watson Research Center \\ Yorktown Heights, N.Y. USA
}

\section{Abstract}

Algebraic semantics is a methodology in theoretical computer science that draws discipline and concepts from universal and categorical algebra. This approach has its roots in a research activity, known as ADJ, which started at IBM Research, Yorktown Heights in the mid Seventies.

In this talk I will discuss the ADJ effort and some of the concepts that have emerged and proved to be important mathematically and for computer science applications. I will stress the common algebraic ideas that span diverse subjects like factorization systems, programming language semantics, continuous algebras and abstract data types. 